\title{
Vergleich der Qualitätsmessung nach §137 SGB V mit Messungen aus Routinedaten
}

\section{S. Drösler

\author{
Signifikante Unterschiede gleicher \\ Indikatoren
}

Qualitätsmanagement

Schlüsselwörter

Qualitätsindikatoren

Routinedaten

Qualitätssicherung

Keywords

quality indicators

hospital administrative data

quality assurance
Institut

Hochschule Niederrhein, Kompetenzzentrum Routinedaten im Gesundheitswesen, Krefeld

Bibliografie

DOI $10.1055 / \mathrm{s}-0031-1286087$

Dtsch Med Wochenschr 2011;

136: S58 - (c) Georg Thieme

Verlag KG Stuttgart · New York .

ISSN 0012-0472

Korrespondenz

Prof. Dr. med. Saskia E. Drösler Hochschule Niederrhein, Kompetenzzentrum Routinedaten im Gesundheitswesen Reinarzstraße 49 47805 Krefeld

Tel. 02151/822-6643

eMail saskia.droesler@

hs-niederrhein.de
Analysiert wurden Daten, die im Jahr 2007 bundesweit im Rahmen der externen Qualitätssicherung nach §137 SGB V erfasst wurden, und eine 10\%-Stichprobe der „Diagnosis Related Groups“ (DRG)-Datenbasis des statistischen Bundesamtes aus dem gleichen Jahr. Es wurde darauf geachtet, dass die jeweiligen Nennerpopulationen der einzelnen Indikatoren exakt miteinander vergleichbar sind. Für alle Indikatoren finden sich statistisch signifikante Unterschiede der jeweiligen Raten. Bemerkenswert ist, dass die Diskrepanzen nicht konsistent sind: Für die Indikatoren geburtshilfliche Verletzungen ( $p<0,01)$, postoperative tiefe Beinvenenthrombose, postoperative Lungenembolie und Letalität bei ambulant erworbener Pneumonie sind die auf Routinedaten basierenden Raten höher $(p<0,001)$. Dagegen liefert der Indikator Dekubitus höhere Raten in der separaten Datenerhebung zur externen Qualitätssicherung nach $\S 137$ SGB V $(p<0,001)$. Für die beobachteten Diskrepanzen werden einerseits eine unzureichende Kodierung der Nebendiagnose Dekubitus sowie andererseits eine übermäßig häufige Berücksichtigung der bei der Krankenhausaufnahme bereits vorliegenden Begleiterkrankungen tiefe Beinvenenthrombose und Lungenembolie vermutet [1].

\section{Methodisches Verbesserungspotential}

Qualitätsindikatoren aus Routinedaten der stationären Versorgung in Deutschland sind aus methodischer Sicht zu verbessern: Bei der Datenerhebung sollte berücksichtigt werden, ob eine Nebendiagnose bereits bei Krankenhausaufnahme vorhanden und somit nicht als Komplikation zu werten ist. International haben zahlreiche Länder diese Kennzeichnung - „present on admission" - bereits umgesetzt und erhalten validere Ergebnisse [2]. Für Qualitätsvergleiche einzelner Indikatoren zwischen Institutionen sollten Methoden erarbeitet werden, die außer den Informationen zu Alter und Geschlecht auch das individuelle Risiko eines Falles, eine relevante Komplikation zu erlangen, berücksichtigen. Darüber hinaus sollte, sofern kritische Ereignisse durch Nebendiagnosen definiert sind und hierdurch erfasst werden, ein möglicherweise unterschiedlicher Dokumentationsumfang, gemessen an der durchschnittlichen Anzahl der dokumentierten Nebendiagnosen der jeweiligen Nennerpopulation, in die Analyse eingehen. Untersuchungen hierzu, die im Rahmen des „Health Care Quality Indicators Project“ der „Organisation for Economic Co-operation and Development" (OECD) durchgeführt wurden, zeigen auffällige Veränderungen der Rankings einzelner Länder nach einer entsprechenden Adjustierung [3].

Autorenerklärung: Es bestehen keine finanziellen Interessenkonflikte in Zusammenhang mit diesem Artikel.

Literatur

1 Maass C, Schleiz W, Weyermann M, Drösler SE. Krankenhaus-Routinedaten zur externen Qualitätssicherung? Vergleich von Qualitätsindikatoren anhand der Daten der gesetzlichen externen Qualitätssicherung (BQS) und Routinedaten. Dtsch Med Wochenschr 2011; 136: 409-414

2 Romano PS, Utter G, Sadeghi B, Zrelak PA, Tancredi $D J$, Baron R, Drösler S, Geppert J. Positive predictive value and potential preventability of the AHRQ patient safety indicators in a national sample of hospitals from the USA. Bulletin The International Society for Quality in Health Care Ltd. 2010

3 Drösler SE, Romano PS, Tancredi DJ, Klazinga NS. International Comparability of Patient Safety Indicators in 15 OECD Member Countries: A Methodological Approach of Adjustment by Secondary Diagnoses. Health Services Research 2011; DOI: 10.1111/j.1475-6773.2011.01290.x 